\title{
Investigation of Exposure Factors for Various Breast Composition and Thicknesses in Digital Screening Mammography Related to Breast Dose
}

\author{
Khaled Alkhalifah Ajit Brindhaban \\ Department of Radiologic Sciences, Faculty of Allied Health Sciences, Kuwait University, Sulaibikhat, Kuwait
}

\section{Significance of the Study}

- The results of this study provide X-ray exposure factors required to acquire optimal diagnostic quality images of breast tissue with different tissue compositions and thicknesses in digital mammography, leading to a better diagnosis of breast cancer manifesting in the form of microcalcifications and mass lesions.

\section{Keywords}

Digital screening mammography · Mammography phantom · Exposure factors · Breast composition · Target/ filter

\begin{abstract}
Objective: To investigate the effect of exposure factors used in digital screening mammography on image quality of different breast compositions. Material and Methods: A digital mammography unit, with tungsten (W) as target, rhodium (Rh) and silver (Ag) as filters, and amorphous selenium detectors, was used to image Computerized Imaging Reference Systems (CIRS) Model 12A phantoms of thickness 4, 5, and 6 $\mathrm{cm}$. Images of each phantom were obtained using targetfilter combinations of $\mathrm{W} / \mathrm{Rh}$ and $\mathrm{W} / \mathrm{Ag}$, at 28,30 , and $32 \mathrm{kVp}$. Images were evaluated by 5 senior technologists with experience in mammography. Image scores were assigned, for
\end{abstract}

each type of feature present in the phantom. Statistical analysis was performed using nonparametric tests to compare sets of image scores at $p=0.05$. Results: A small but statistically significant improvement was detected in the visibility of microcalcifications $(8.8 \pm 0.2 ; p=0.031)$ for the W/Rh combination but this did not show any differences in the visibility of masses or fibers. The entrance skin dose (ESD) and mean glandular dose (MGD) were lower for the W/Ag (ESD = 1.30-3.70; MGD = 0.44-0.93 $\mathrm{mGy}$ ) combination compared to W/Rh (ESD = 1.66-5.40; MGD = 0.52-1.12 mGy). The MannWhitney test revealed that $30-k V$ exposure with the W/Rh combination showed a significantly better visibility of specks in the 30/70 phantom compared to other exposures. Conclusion: The use of an Rh filter showed a better image quality for all phantoms. 28 and $30 \mathrm{kVp}$ with the W/Rh combination provided a slightly better image quality, and the MGD is less than $1.2 \mathrm{mGy}$.

(c) 2018 The Author(s)

Published by S. Karger AG, Basel

\begin{tabular}{|c|c|c|}
\hline KARGER & $\begin{array}{l}\text { (c) } 2018 \text { The Author(s) } \\
\text { Published by S. Karger AG, Basel }\end{array}$ & $\begin{array}{l}\text { Karger } \\
\text { Open access }\end{array}$ \\
\hline $\begin{array}{l}\text { E-Mail karger@karger.co } \\
\text { www.karger.com/mpp }\end{array}$ & $\begin{array}{l}\text { This is an Open Access article license } \\
\text { Attribution-NonCommercial-4.0 Int } \\
\text { (http://www.karger.com/Services/Op } \\
\text { the online version of the article or } \\
\text { commercial purposes requires writte }\end{array}$ & $\begin{array}{l}\text { the Creative Commons } \\
\text { al License (CC BY-NC) } \\
\text { ssLicense), applicable to } \\
\text { ge and distribution for } \\
\text { ission. }\end{array}$ \\
\hline
\end{tabular}

Dr. Khaled Al Khalifah

Department of Radiologic Sciences, Faculty of Allied Health Sciences, Kuwait University PO Box 31470

Sulaibikhat 90805 (Kuwait)

E-Mail k_alkhalifah@hsc.edu.kw 


\section{Introduction}

Breast cancer is one of the most commonly diagnosed malignancies in American women and the second leading cause of cancer deaths in women [1]. Over the last 30 years, the incidence of breast cancer among the Kuwait population has increased threefold from 15 to 50 cases/100,000 population [2]. In many studies the combination of a clinical breast examination and screening mammography has increased breast cancer detection rates and the number of cancers detected at more localized stages, thus lowering the rate of breast cancer deaths [3-7]. Most mammographic systems on the market have the ability to automatically select a beam quality based on compressed breast tissue thickness. They also allow radiographers to manually set tube potential and target filter combinations [8]. Optimization of exposure parameters in digital mammography is necessary to maximize the contrast-to-noise ratio (CNR) of the image, while simultaneously minimizing patient dose [8]. Since the breast is a highly radiosensitive organ, it is important that the dose level during screening mammography be kept as low as possible so that screening itself does not increase the risk of breast cancer induction $[9,10]$. Image quality and the probability of relevant anatomical details or pathology displayed is affected by the amount of tissue coverage, contrast between tissues, exposure latitude or dynamic range, spatial resolution of the imaging system, any noise, and artifacts present in the image [11]. Image quality depends critically on the design and performance of the radiographic unit, the type of image receptor and the manner in which the equipment is used to acquire and process the mammogram. In addition, the type of display and the conditions under which the image is viewed have an important effect on the ability of the radiologist to extract the information recorded in the mammogram [12].

The composition of breast tissue can vary from mostly adipose to variable quantities of radiologically dense tissue, due to patient age and genetic factors [10]. A large variety of breast phantoms have been produced with tissue-equivalent materials and used in an attempt to simulate living organs in terms of attenuation, density of tissue, and the radiographic visibility [13]. Previous studies had shown that a fully digital mammography (FDM) system has optimum image quality and is capable of detecting subtle calcification clusters with low dose [14, 15]. Aminah et al. [8] reported potential for dose reduction up to $11 \%$ for a set CNR of 3.0 by using beam quality other than that determined by automatic exposure control selection. This study also found that exposure factors for optimal image quality depended on the composition (glandular-to-adipose) of breast tissue. However, the dependence of exposure factors on different thicknesses of breast tissue has not been studied widely.

Hence, the aim of our study was to investigate the effect of exposure factors used in digital screening mammography on detection of masses and microcalcifications on different tissue compositions and thickness.

\section{Materials and Methods}

\section{Breast Phantom Model}

A Hologic Selenia (Hologic Inc., Marlborough, MA, USA) digital mammography (DM) unit, with a tungsten (W) target, with dual filter materials rhodium (Rh) and silver $(\mathrm{Ag})$, was used in this study for imaging the phantoms. The unit is equipped with amorphous selenium detectors of $70-\mu \mathrm{m}$ pixel size. There different phantoms of the Computerized Imaging Reference Systems (CIRS, Norfolk, VA, USA) model 12A, which include tissue-equivalent phantoms of 4,5 , and $6 \mathrm{~cm}$ thickness, were used to mimic breast tissues of different compositions. The glandular content of each phantom is 50,30 , and $20 \%$, respectively. They contain 5 nylon fibers of diameters $1.25,0.83,0.71,0.53$, and $0.30 \mathrm{~mm}$, respectively; 7 simulated spherical tumor masses of diameter 4.76, 3.16, 2.38, $1.98,1.59,1.19$, and $0.90 \mathrm{~mm}$, respectively; 12 groups of microcalcifications, placed at different locations within the phantom with each group containing 6 microcalcifications of diameters ranging from 0.400 to $0.130 \mathrm{~mm}$ (Fig. 1).

\section{Image Acquisition and Data Collection}

Three images were obtained using each phantom and targetfilter combination (W/Rh and $\mathrm{W} / \mathrm{Ag}$ ), one each at 28, 30, and 32 $\mathrm{kVp}$, respectively. These kilovolt peak values represent the range used in clinical studies with patients. The automatic exposure control unit of the system was used to determine the appropriate milliampere-seconds for all images. The entrance surface dose (ESD) and mean glandular dose (MGD) were recorded from the Hologic Selenia DM unit for each exposure. The images were processed according to the manufacturer-recommended methods [6].

\section{Reader Study}

All images were evaluated by 5 senior technologists, with average work experience of 15 years (range: 12-20 years) who work in the screening mammography centers using Barco's (Barco Inc., Zug, Switzerland) 5-megapixel display systems for digital breast imaging. The reviewers were prohibited from making any further enhancements, such as adjusting window level and/or window width, during their evaluation. They were allowed sufficient time to view the images and were requested to write down the number of groups of microcalcifications, number of fibers, and number of masses that were visible. Image scores were assigned, for each type of feature present in the phantoms, equal to the number of features visible to the observers.

\section{Statistical Analysis}

Statistical analysis was performed using the Mann-Whitney test for comparing 2 sets of scores and the Kruskal-Wallis test for 
Fig. 1. CIRS mammography research phantom model 12A.

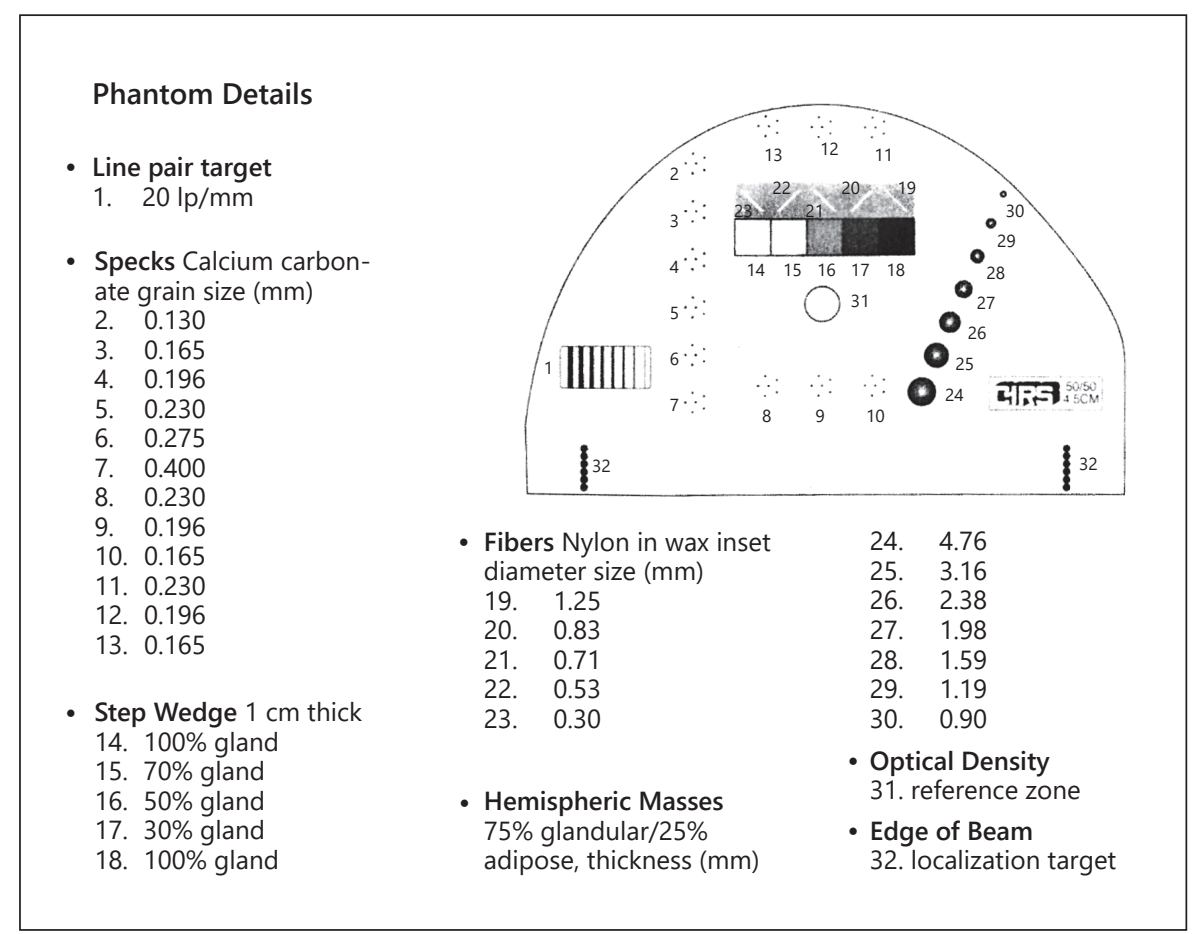

Table 1. MGD and ESD with various kilovolt peaks $(\mathrm{kVp})$ for phantom $50 / 50$

\begin{tabular}{lllll}
\hline Target/filter & $\mathrm{kVp}$ & $\mathrm{mAs}$ & $\mathrm{MGD}, \mathrm{mGy}$ & $\mathrm{ESD}, \mathrm{mGy}$ \\
\hline W/Rh & 28 & 51 & 0.61 & 2.12 \\
W/Rh & 30 & 40 & 0.60 & 1.98 \\
W/Rh & 32 & 29 & 0.52 & 1.66 \\
W/Ag & 28 & 33 & 0.51 & 1.66 \\
W/Ag & 30 & 26 & 0.50 & 1.60 \\
W/Ag & 32 & 18 & 0.44 & 1.30 \\
\hline
\end{tabular}

comparing more than 2 sets of scores at the $p=0.05$ level, with the aid of the software package Statistical Package for Social Sciences (SPSS, IBM SPSS Statistics for Windows, version 22.0, IBM Corp., Armonk, NY, USA).

\section{Results}

The exposure factors MGD and ESD for the 3 different exposures of the phantoms with 50,30 , and $20 \%$ glandular content are shown in Tables 1-3, respectively. The MGD and ESD for W/Ag were lower than W/Rh in all 3 phantoms. The number of visible features for the 3 exposures of the phantoms with 50, 30, and $20 \%$ glandular content are illustrated in Figures 2-4, respectively. Statis-
Table 2. MGD and ESD with various kilovolt peaks $(\mathrm{kVp})$ for phantom $30 / 70$

\begin{tabular}{lllll}
\hline Target/filter & $\mathrm{kVp}$ & $\mathrm{mAs}$ & $\mathrm{MGD}, \mathrm{mGy}$ & $\mathrm{ESD}, \mathrm{mGy}$ \\
\hline W/Rh & 28 & 75 & 0.77 & 3.19 \\
W/Rh & 30 & 58 & 0.76 & 2.97 \\
W/Rh & 32 & 45 & 0.72 & 2.69 \\
W/Ag & 28 & 46 & 0.65 & 2.36 \\
W/Ag & 30 & 35 & 0.62 & 2.20 \\
W/Ag & 32 & 29 & 0.60 & 2.14 \\
\hline
\end{tabular}

Table 3. MGD and ESD with various kilovolt peaks $(\mathrm{kVp})$ for phantom 20/80

\begin{tabular}{llrll}
\hline Target/filter & $\mathrm{kVp}$ & $\mathrm{mAs}$ & $\mathrm{MGD}, \mathrm{mGy}$ & $\mathrm{ESD}, \mathrm{mGy}$ \\
\hline W/Rh & 28 & 122 & 1.12 & 5.40 \\
W/Rh & 30 & 91 & 1.10 & 4.80 \\
W/Rh & 32 & 69 & 0.98 & 4.20 \\
W/Ag & 28 & 69 & 0.93 & 3.70 \\
W/Ag & 30 & 51 & 0.80 & 3.30 \\
W/Ag & 32 & 38 & 0.77 & 2.90 \\
\hline
\end{tabular}




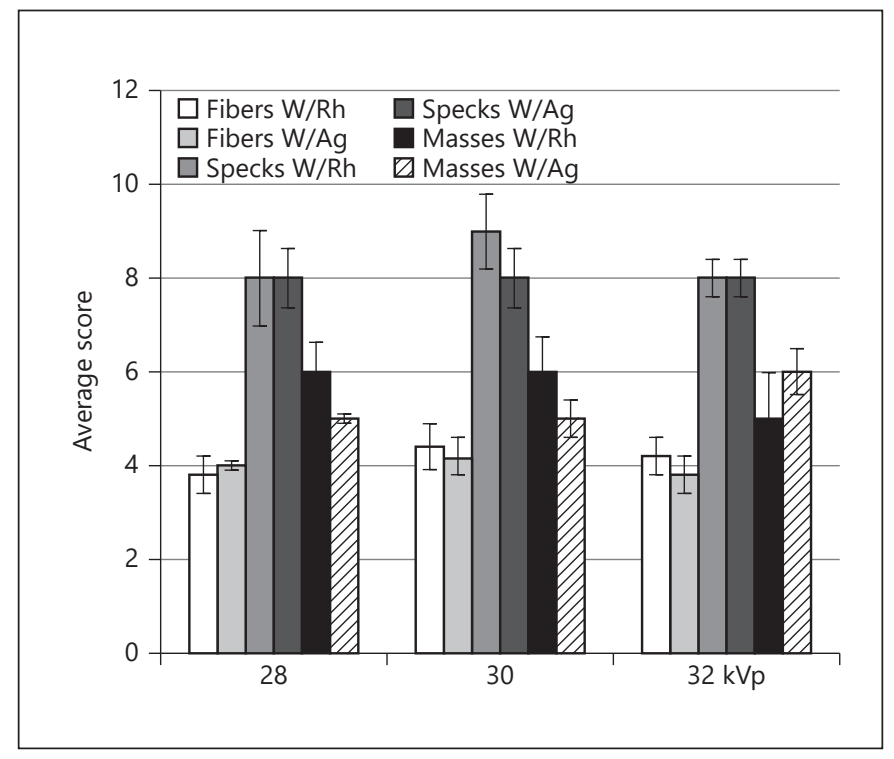

Fig. 2. The average score for each feature with different kilovolt peaks for the 50/50 phantom.

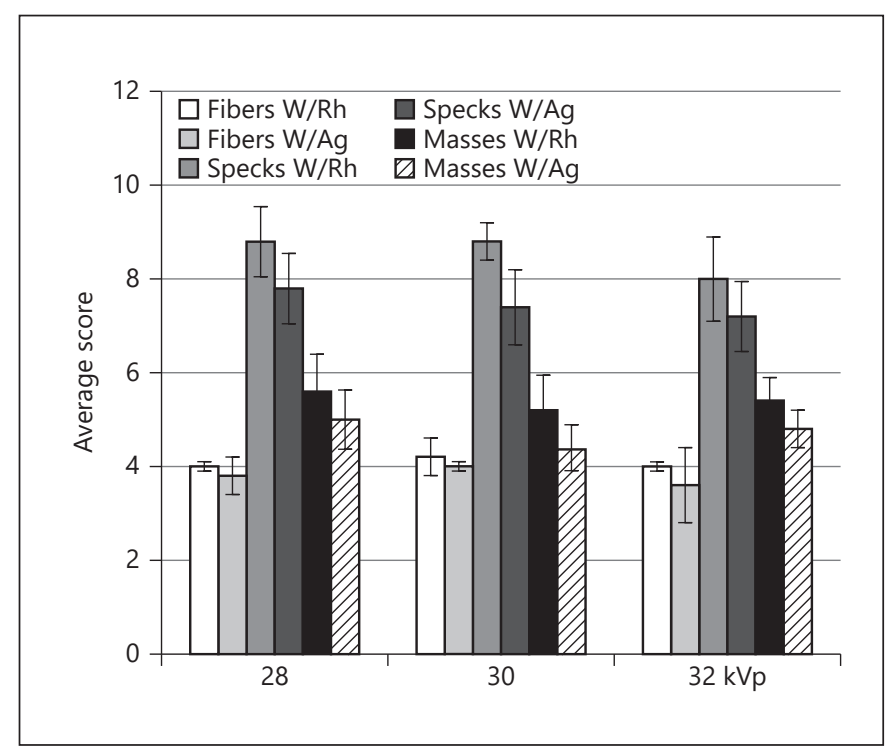

Fig. 3. The average score for each feature with different kilovolt peaks for the $30 / 70$ phantom.

tically significant differences were not observed for any of the 3 features in any of the 3 phantoms when exposure factors were changed, except for the visibility of microcalcifications in the phantom with $30 \%$ glandular content $(p=0.031)$. The Mann-Whitney test revealed that the 30$\mathrm{kVp}$ exposure with the $\mathrm{W} / \mathrm{Rh}$ combination showed a sig-

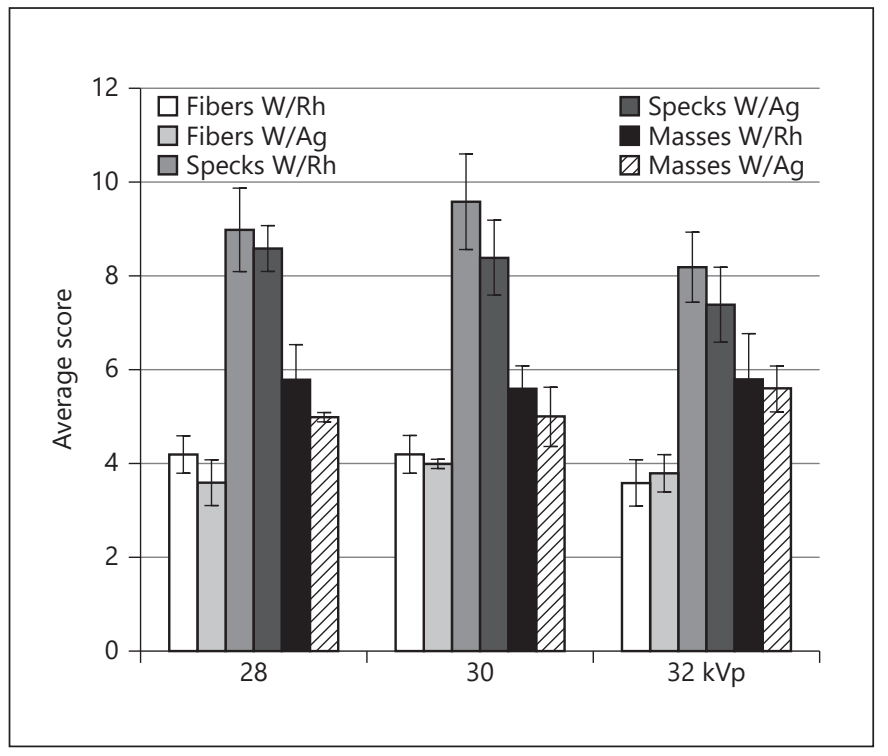

Fig. 4. The average score for each feature with different kilovolt peaks for the $20 / 80$ phantom.

nificantly better visibility of specks in this phantom compared to other exposures.

Statistically significant differences were not observed between specific kilovolt peaks and filters used for the $50 / 50$ phantom, $30 / 70$ phantom and $20 / 80$ phantom, but in sum of ranks the Rh filter provided a better detectability for fibers, microcalcifications, and masses for all kilovolt peak values used in this study. However, for the 50/50 phantom, the image acquired at $30 \mathrm{kVp}$ with the $\mathrm{W} / \mathrm{Rh}$ combination produced the highest mean rank for the visibility of fibers. For the visibility of microcalcifications 28 and $30 \mathrm{kVp}$ with the $\mathrm{W} / \mathrm{Rh}$ combination and $32 \mathrm{kV}$ with the W/Ag combination resulted in the higher mean rank values than other combinations. For masses, the $28 \mathrm{kVp}$ with $\mathrm{W} / \mathrm{Rh}$ exposure resulted in the highest mean rank. For the 30/70 phantom, the optimum kilovolt peak which resulted in the highest mean rank was 30 for fibers, 28 for microcalcifications, and for the masses it was 28 and 32 $\mathrm{kVp}$ with W/Rh. For the $20 / 80$ phantom, 28 and $32 \mathrm{kVp}$ are optimal for fibers, $30 \mathrm{kVp}$ for specks, and $28 \mathrm{kVp}$ for masses, all for the $\mathrm{W} / \mathrm{Rh}$ combination.

\section{Discussion}

Our current work indicated that the use of the W/Rh combination may result in a slightly better image quality than the W/Ag combination for different breast tissue 
compositions. One previous study [8] reported that as the breast thickness increases, there is a greater possibility for dose reduction using $\mathrm{W} / \mathrm{Rh}$ followed by $\mathrm{Mo} / \mathrm{Rh}$ and $\mathrm{Mo} /$ Mo combinations. Another study [16] recommended 28, 29 , and $31 \mathrm{kVp}$ without comparing between $\mathrm{W} / \mathrm{Rh}$ and $\mathrm{W} / \mathrm{Ag}$, but supported the idea that using the W/Rh combination results in a reduction of dose.

The detectability of different structures of the human breast tissue in a radiographic image is influenced by several image quality parameters. Three of these - subject contrast, spatial resolution of the imaging system, and image noise - have been commonly used to assess image quality. The subject contrast is influenced by the X-ray beam quality, which depends on the kilovolt peak setting and the target/filter combination. Higher X-ray beam qualities reduce subject contrast. Image noise obscures structures and affects detectability of structures. Since the $\mathrm{Rh}$ filter removes from the X-ray beam the components above its k-edge, the average energy of the beam is slightly lower than when Ag was used as a filter. The higher beam quality from the Ag filter results in lower subject contrast and hence slightly lower detectability of features in the phantom. In FDM, some loss of subject contrast due to high X-ray beam quality can be recovered using image enhancement techniques built into the imaging systems. Quantum noise and anatomical noise are present in all images. Quantum noise, which obscures small structures like microcalcifications and fibers, can be reduced by increasing the amount of X-rays used to form the image. Anatomical noise, which affects the detectability of larger structures like masses, mostly depends on the imaging geometry rather than X-ray beam quality. The contribution from quantum noise to the total image noise is much higher than that from the anatomical noise. These reasons could be used to explain the lack of statistically significant differences in the detectability of structures when kilovolt peak and filter material were changed in our study $[17,18]$.

The reduction in MGD and ESD when the W/Ag combination was used could be attributed to the higher X-ray beam quality, leading to a larger proportion of the beam penetrating the breast tissue and reaching the image receptor. The optimization principle of radiation protection requires that diagnostic quality images be acquired using the least possible radiation dose to the patient. The European Commission has set a guidance MGD level of $1.3 \mathrm{mGy}$ for mammography examinations of a standard breast. All the exposure factors used in our study resulted in MGD less than $1.2 \mathrm{mGy}$ and in some cases much lower values.

Exposure Factors in Digital Screening

Mammography
There are some limitations to our study which should be considered when interpreting the results. Firstly, this study was conducted on a single FDM system and therefore the results may vary slightly when other FDM systems are used. Further studies involving multiple FDM systems are proposed. Secondly, image quality analysis in this study was carried out subjectively by observers, and the results may vary if a different group of observers were used. Although interobserver variability was not analyzed in this study, the error bars (1 SD) indicated in Figures 2-4 represent a relatively small spread of observer responses to all 3 features. Interobserver variability can be minimized if objective methods of image analysis, such as measuring signal-to-noise ratio and/or CNR, are used. Although signal-to-noise ratio and CNR are very useful image quality measures for assessing imaging system performance, it may be of less relevance in clinical situations. The authors chose to use observers because it closely resembles the clinical situation where radiologists report on mammographic images.

\section{Conclusion}

The use of the Rh filter showed better image quality for all phantoms. The authors recommend the use of 28 and $30 \mathrm{kVp}$ as exposure factors with the W/Rh combination for screening DM. These factors resulted in MGD less than $1.2 \mathrm{mGy}$ which meets the international standards.

\section{Acknowledgments}

I would like to thank the Ministry of Health to use the mammographic machine, and special thanks go to all senior technologists for their help with evaluating the images.

References $\nabla_{1}$ Ho JM, Jafferjee N, Covarrubias GM, et al: Dense Breasts: A review of reporting legislation and available supplemental screening options. Am J Roentgenol 2014;203:449-456.

-2 Elbasmi A, Al-Asfour A, Al-NesfY, et al: Cancer in Kuwait: magnitude of the problem. Gulf J Oncol 2010;8:7-14.

- 3 Swedish Organised Service Screening Evaluation Group: Reduction in breast cancer mortality from organized service screening with mammography: further confirmation with extended data. Cancer Epidemiol Biomarkers Prev 2006;15:45-51. 
4 Tabár L, Vitak B, Chen HH, et al: Beyond randomized controlled trials: organized mammographic screening substantially reduces breast carcinoma mortality. Cancer 2001;91: 1724-1731.

5 Tabár L, Yen MF, Vitak B, et al: Mammography service screening and mortality in breast cancer patients: 20-year follow-up before and after introduction of screening. Lancet 2003; 36:1405-1410.

-6 Pauwels EKJ, Foray N, Bourguignon MH: Breast cancer induced by X-ray mammography screening? A review based on recent understanding of low-dose radiobiology. Med Princ Pract 2016;25:101-109.

7 Gupta R, Nayak M, Khoursheed M, et al: Pain during mammography: impact of breast pathologies and demographic factors. Med Princ Pract 2003;12:180-183.

8 Aminah M, Ng KH, Abdullah BJJ, et al: Optimal beam quality selection based on contrastto-noise ratio and mean glandular dose in digital mammography. Australas Phys Eng Sci Med 2010;33:329-334.
-9 Chawla AS, Samei E, Saunders R, et al: Effect of dose reduction on the detection of mammographic lesions: a mathematical observer model analysis. Med Phys 2007;34:33853397.

10 Bushberg JT, Seibert JA, Leidholdt JEM, et al: The Essential Physics of Medical Imaging. Philadelphia, Lippincott Williams \& Wilkins, 2002.

11 Kanal KM, Krupinski E, Berns EA, Geiser WR, et al: ACR-AAPM-SIIM practice guidelines for determinants of image quality in digital mammography. J Digit Imaging 2013;26: 10-25.

12 Yaffe MJ, Bunch PC, Desponds L, et al: Technical aspects of image quality in mammography. J ICRU 2009;9:33-51.

13 De Almeida CD, Coutinho CMC, Peixoto JE, et al: Study of attenuation coefficient of a breast phantom used in diagnostic radiology. International Nuclear Atlantic Conference INAC 2009, Rio de Janeiro, 2009.
4 Slusarczyk-Kacprzyk W, Skrzynski W, Fabiszewska E: Evaluation of dose and image quality in mammography with screen-film, CR, and DR detectors - application of the ACR Phantom. Polish Radiol 2016;18:386391.

15 Warren LM, Mackenzie A, Cooke J, et al: Effect of image quality on calcification detection in digital mammography. Med Phys 2012;39: 3202-3213.

16 Kamal I, Kumari K, Vijayalakshimi K, et al: Determination of tube output $(\mathrm{kVp})$ and $\mathrm{ex}$ posure mode for breast phantom of various thicknesses/glandularity for digital mammography. Malays J Med Sci 2015;22:40-49.

17 Tischenko O, Hoeschen C, Buhr E: Reduction of anatomical noise in medical X-ray images. Rad Prot Dosim 2005;114:69-74.

18 Sen A, Gifford HC: Accounting for anatomical noise in search-capable model observers for planer nuclear imaging. SPIE J Med Imag 2016;3:1-10. 